\title{
Electromagnetic turbulent structures: an ubiquitous feature of the edge region of toroidal plasma configurations
}

M. Spolaore ${ }^{1}$, N. Vianello ${ }^{1}$, I. Furno ${ }^{2}$, D. Carralero ${ }^{34}$, M. Agostini ${ }^{1}$, J. A. Alonso ${ }^{3}$, F. Avino $^{2}$, R. Cavazzana ${ }^{1}$, G. De Masi ${ }^{1}$, A. Fasoli ${ }^{2}$, C. Hidalgo ${ }^{3}$, E. Martines ${ }^{1}$, B. Momo ${ }^{1}$, A. Scaggion ${ }^{1}$, P. Scarin ${ }^{1}$, S. Spagnolo ${ }^{1}$, G. Spizzo ${ }^{1}$, C. Theiler ${ }^{2}$, M. Zuin ${ }^{11}$

1 Consorzio RFX, Corso Stati Uniti 4,35127 Padova, Italy

2 Centre de Recherches en Physique des Plasmas (CRPP),

Ecole Polythechnique Fédérale de Lausanne (EPFL), CH-1015 Lausanne,

Switzerland

${ }^{3}$ Laboratorio Nacional de Fusion, CIEMAT, Avda. Complutense 40, 28040 Madrid, Spain

${ }^{4}$ Max Planck Institute for Plasma Physics, Boltzmannstr.2,85748 Garching, Germany ${ }^{\mathrm{a}}$

(Dated: 5 November 2014)

Electromagnetic features of turbulent filaments, emerging from a turbulent plasma background, have been studied in four different magnetic configurations: the stellarator TJ-II, the Reversed Field Pinch RFX-mod, a device that can be operated also as a ohmic tokamak, and the Simple Magnetized Torus, TORPEX. By applying an analogous diagnostic concept in all cases, direct measurements of both field-aligned current density and vorticity were performed inside the filament. The inter-machine comparison reveals a clear dependence of the filament vorticity upon the local timeaveraged $E \times B$ flow shear. Furthermore, a wide range of local beta was explored allowing concluding that this parameter plays a fundamental role in the appearance of filament electromagnetic features.

PACS numbers: 52.25.Xz, 52.35.Ra, 52.35.We, 52.70.Ds

\section{Accepted for publication 21 November 2014 \\ Physics of Plasmas 22, 012310 (2015); \\ doi: http://dx.doi.org/10.1063/1.4906869}

${ }^{a)}$ Electronic mail: monica.spolaore@igi.cnr.it 
Electromagnetic turbulent structures

\section{INTRODUCTION}

Plasma blobs or field-aligned filamented structures have been detected in magnetized plasmas ranging from laboratory, including thermonuclear fusion devoted experiments ${ }^{1}$, to astrophysical ones ${ }^{2,3}$. They provide a privileged channel for particle, energy and momentum losses conveyed in magnetized plasmas towards the wall, so that their study represent a hot topic in fusion plasmas ${ }^{4,5}$. In particular electrostatic (ES) features of filaments have been widely studied both from theoretical and experimental point of view, as described in the review papers ${ }^{6,7}$. The description of blobs, since their first experimental observation ${ }^{1}$, is usually performed in the plane perpendicular to the local magnetic field, but in recent years an increasing interest also on their parallel structure came out ${ }^{8,9}$. A particular focus of the present paper is the structure of filaments in the direction parallel to the magnetic field, this extends previous investigations on blob perpendicular dynamics ${ }^{9}$.

In recent years an increasing theoretical and experimental interest have raised $^{7}$ for the filament electromagnetic (EM) features. This interest arises first of all because future devices are expected to operate eventually at higher $\beta$ where electromagnetic effects could play a role in the dynamics of blobs and filaments ${ }^{10}$. Furthermore, in the case of macroscopic events like the ELMs, a large amount of current has been observed to be conveyed in the SOL by ELM filaments ${ }^{11}$. This suggests that electromagnetic filaments could be responsible also for bending magnetic field lines ${ }^{10}$, local modification of magnetic topology ${ }^{12}$ or wider modification of the plasma column as suggested for example in the case of ASDEX-Upgrade. Here it has been found that the ELMs are associated with a reduction of the plasma radius hinting to a movement of the plasma outside the last close flux surface and to a redistribution of the edge current profile ${ }^{13}$. In the present paper we focus in particular on the direct measurement of turbulent filamentary structures current density, which can provide an efficient channel for current transport ${ }^{14}$. In this respect, it has been found that the amount of the current density can play a role in the filament radial velocity ${ }^{15,16}$ and ultimately in the transport of the advected plasma. From all these considerations it results that understanding electromagnetic filamentary transport still represents a fundamental topic in the fusion energy research panorama.

The simplest theoretical description of a plasma filaments is based on an equivalent electric circuit of the plasma structure. This includes a driving term for the current which 
provides a charge separation,

usually represented by an equivalent gravity force. A common driving mechanism is represented by the magnetic curvature, which induces a parallel current dipole in the cross-field plane. The parallel current arises to ensure quasi-neutrality in response to the charge polarization, and along the field line closes the loop, for example, on the divertor plates or limiter, where sheath boundary conditions apply ${ }^{17}$. Apart from this explanation, strongly localized current filaments could appear for different reasons, as for example because of the coupling of drift and Alfvén waves which have been suggested to occur at the tokamak edge in particular at higher $\beta$ values ${ }^{18}$. From the experimental point of view, the presence of electromagnetic features on fusion relevant edge plasma filaments were deduced from local measurement of magnetic fluctuations formerly on MAST device ${ }^{1}$, although previous experimental investigations have been performed also in linear devices ${ }^{19}$. More recently a direct observation of current density filaments associated to turbulent structures has been performed in the edge region of the RFX-mod reversed field pinch (RFP) device ${ }^{8}$, where drift kinetic Alfvén structures have been identified ${ }^{20}$. Similar EM filaments have been observed also in the TORPEX experiment ${ }^{21}$, simple magnetized toroidal plasma, where for the first time the parallel current density cross-field map in the filaments, originated by ideal interchange waves, was experimentally revealed.

The present paper extends the understanding and characterization of current-carrying blobs, by providing experimental characterization of these structure in four different magnetic configurations. The data have been collected using the same diagnostic concept, specifically developed and applied for each configuration. Electromagnetic characterization of the blobs is provided up to the 3D details and allowing to extrapolate the same fundamental quantities, parallel current density and vorticity in primis, for a complete inter-machine scaling. The obtained scaling has allows to disentangle the role of local electron $\beta$ and flow shear, both quantities explored over a wide range, in determining the dynamics, energetics and characteristics of the blob. The present approach represents a unique attempt to provide information on small scale fluctuations to disentangle the basic physical picture regardless of the peculiar magnetic configuration.

The data presented hereafter are obtained in toroidal magnetic confinement devices including TORPEX, characterized by open field lines, and the hotter plasmas of fusion devoted experiments as TJ-II stellarator and RFX-mod, which can be operated with both RFP and 
Electromagnetic turbulent structures

ohmic tokamak configurations. The paper is organized as follows: experiments and diagnostic concept are described in section II; in section III the main experimental findings in RFP RFX-mod, tokamak RFX-mod, stellarator TJ-II and simple magnetized torus (SMT) TORPEX are shown. In section IV, the experimental results are summarized and discussed in terms of inter-machine comparison.

\section{EXPERIMENTAL SETUP AND DIAGNOSTIC METHODS}

Data presented hereafter are collected in three different devices, allowing for exploring totally four different magnetic configuration.

The TJ-II is a medium size heliac stellarator located in Madrid, Spain, ${ }^{22}$, major radius, $R=1.5 \mathrm{~m}$ and average minor radius $\langle a>=0.22 \mathrm{~m}$. The rotational transform at the separatix is $\iota(a) / 2 \pi=1.6$ and the nominal magnetic field is $B_{t}=1 \mathrm{~T}$. The device is equipped with additional heating systems: ECRH $200-400 \mathrm{~kW}$ and NBI maximum nominal power 1 MW. The simple magnetized experiment TORPEX ${ }^{23}$, located in Lausanne, Switzerland, is a toroidal device ( $R=1 \mathrm{~m}$ and $a=0.2 \mathrm{~m}$ ), where the plasma is produced by injecting microwave power $\left(P_{E C} \approx 400 \mathrm{~W}\right)$ at the electron cyclotron resonance frequency. The magnetic configuration is obtained by superimposing a vertical field $B_{v}=1.6 \mathrm{mT}$ and a toroidal one $B_{t}=76 \mathrm{mT}$, resulting in helical magnetic field lines with curvature and $\nabla B$, which end on top and bottom sides of the vacuum vessel. RFX-mod, located in Padova, Italy, is the largest Reversed Field Pinch device $(R=2 \mathrm{~m}$ and $a=0.459 \mathrm{~m})$ and can operate up to $I_{p}=2 \mathrm{MA}$ plasma current ${ }^{24}$. The maximum toroidal magnetic field is $B_{t}=0.7 \mathrm{~T}$. Recently $^{25}$ the RFX-mod device was also operated as a ohmic tokamak with $I_{p}<0.2 \mathrm{MA}$, and in particular the first results obtained with insertable probes in such circular ohmic tokamak discharges are here presented. To obtain simultaneous measurements of both electrostatic (ES) and electromagnetic (EM) fluctuations and to facilitate the comparison among the different devices, we apply the same diagnostic concept in all the studied plasmas, adapted by specifically designed diagnostics tools to the different plasma environments. In each of the devices, the experiments were conceived for providing ES measurements associated to filaments, such as density, $n_{e}$, temperature, $T_{e}$, pressure, $p_{e}$, and potentials. Apart from these, two other quantities not so commonly available simultaneously are obtained. These are: the filament parallel vorticity, $\omega_{\|}=(\nabla \times \vec{v})_{\|}$, where $\vec{v}$ is the local electric drift flow, 
and the parallel current density estimated from the Ampere's law, $J_{\|}=(\nabla \times B)_{\|} / \mu_{0}$, where $B$ is the local average magnetic field. More in detail, $\omega_{\|}$was obtained from $2 \mathrm{D}$ arrays in the cross-field plane of floating potential, $V_{f}$, measurements, $\omega_{\|} \approx-\nabla_{\perp}^{2} V_{f} / B$, whereas a direct experimental estimate of the $J_{\|}$was obtained by $2 \mathrm{D}$ arrays in a cross-field plane of triaxial magnetic coils.

Historically the first probe conceived for the concurrent investigation of parallel current in a fusion oriented device has been developed for the RFX-mod experiment. This diagnostic, dubbed U-Probe, was built as a simplified 2D version of the one adopted in the Cluster mission for the measurement of currents in magnetosphere is applied ${ }^{26}$. The diagnostic has been vastly described in previous articles ${ }^{8,20}$ and is shown in the respective picture of figure 1: it consists of two boron nitride "towers" spaced in the perpendicular direction along the $E \times B$ edge flow direction, toroidal in the case of the RFP. Electrostatic graphite pins are installed on each of those towers and arranged in 5 rows by 8 radial arrays in the cross-field plane, equally spaced in both directions by $6 \mathrm{~mm}$. These pins provide information on density, temperature and floating potential in different positions. In particular for the present measurements the pins rows are used in the 5-pins triple probe configuration ${ }^{27}$. In addition, in each of those towers a radial array of 7 triaxial pick-up coils is also installed to compute the parallel current directly from the circuitation of the magnetic field. The U-probe head, suitable tilted, has been used for the investigation of the same parameters in the tokamak operation also, providing the first direct measurement of parallel current density associated to filaments in this configuration. In the TORPEX device, a further simplified probe has been realized and the first data obtained have been already presented $i^{21}$. This probe, named L-shaped current probe, consists of triaxial coils located at the vertices of a triangle and laying in the perpendicular plane housed in small quartz-tubes. In the specific case of limiter proximity in TORPEX, $J_{\|}$measurements were carried out also by a Single Sided Langmuir Probe (SSLP) biased at the limiter potential ${ }^{21}$. In TORPEX, further information has been obtained using other diagnostics, which can be combined together thanks also to the excellent reproducibility of the discharges. On TORPEX, noticeable steps are carried on and here presented in order to provide 3D measurements of the parallel current density and vorticity associated to a single filament. Finally in TJ-II the so-called 'vorticity probe', described in detail in ${ }^{28}$, has been used. This probe, similarly to the UProbe, combines both the electrostatic and magnetic measurements into a unique diagnostic: 
Electromagnetic turbulent structures

it computes the parallel current from magnetic field circuitation, where the probes are as in the case of TORPEX displaced in a triangular shape, in the cross-field plane. Vorticity is estimated from floating potential measurements, at spacial points forming a 5-pins cross in the perpendicular plane. The rest of the available electrostatic pins are used for getting information on temperature and density at the probe location, by using the triple probe technique. In figure 1 are shown the pictures of the diagnostic tools, specifically designed for the investigation of the electromagnetic filaments in the different above mentioned devices.

\section{FILAMENT EM EXPERIMENTAL MEASUREMENTS}

\section{A. RFX-mod}

RFX-mod: Reversed Field Pinch RFX-mod Reversed Field Pinch experiment was one of the first fusion relevant plasmas providing direct measurements of the electromagnetic features associated to plasma blobs ${ }^{8}$. We would like here to summarize the main experimental observation already reported in several publications ${ }^{8,20,29}$.

The following measurements refer to hydrogen discharges with a relatively low plasma current, $I_{p} \leq 400 k A$, so that the involved power allows the insertion of probes at the edge. Average density normalized to the Greenwald value is $n / n_{g} \approx 0.4 \div 0,5$ with a reversal parameter $B_{\phi}(a) /\left\langle B_{\phi}\right\rangle \approx-0.07$. The U-probe head was inserted radially from the outer equator and oriented with the plane including the two towers perpendicular to the mainly poloidal magnetic field at edge, as shown in 1. For these measurements the electrostatic pins were used in the 5-pins triple probe configuration to measure density and temperature in the same nominal location and in different radial positions. The local pressure measurement $p_{e}=n_{e} T_{e}$ is then available with high time resolution $5 \mathrm{MHz}$. A complete characterization of the perpendicular electromagnetic features associated with blobs has been obtained in relatively high $\beta$ plasmas $(\approx 1 \%)$, through space-time conversion of radial arrays of single point measurements. The statistical technique adopted to identify coherent structures emerging from turbulent background is based on wavelet analysis. The technique is described in detail in ${ }^{30,31}$. For this paper the mother wavelet used is the Mexican hat, and the use of the Local Intermittency Measure (LIM) is applied for distinguishing on wavelet coefficients the intermittent events, from the Gaussian background. The conditional average 
technique $^{32,33}$ is then applied using as a trigger the appearance at a given time scale of an intermittent event in the reference signal, $p_{e}$ in this case. An example of the conditional average on pressure events detected at $200 \mathrm{kHz}$ time scale is shown in figure 2. A clear peak on pressure is associated to filaments. It has been proven $^{8,20}$ that these blobs are pressure perturbation caused by a local enhancement of the plasma density, with a radial extension of a few local ion sound gyroradii $\rho_{s}$. The plasma potential is found to be nearly in phase with respect to the density, consistent with a drift nature of this structure ${ }^{20}$.

The structures are found to propagate consistently with the main $\mathbf{E} \times \mathbf{B}$ flow, whose radial profile is shown in Fig. 3 (top left panel). The picture shows the main component,the toroidal one, of $\mathbf{E} \times \mathbf{B}$ measured in the RFX-mod RFP configuration in the range $r / a>0.9$ and for reversal parameter $F>-0.05$. The filament propagation behavior has been proved both by electrostatic probes, Gas Puffing Imaging diagnostic ${ }^{34}$ and through the observation of the perpendicular propagation of their magnetic fingerprints ${ }^{20}$. A direct estimate of the vorticity represents an intrinsic 2D information of the blob ${ }^{29}$ and it was found that the vorticity fluctuation is consistent with the reconstructed 2D floating potential map corresponding to a filament. This further confirms the reliability of the underlying frozen turbulent hypothesis ${ }^{35}$, that allows the time-space conversion of single measurement points. This hypothesis is in fact usually applied for the filament detection and for obtaining their average spatial structure via the conditional average technique. Using as a trigger intermittent events in the pressure time-traces allows for reconstructing conditionally sampled quantities associated with filaments. In particular, the complex electromagnetic measurements already described in the previous section has allowed the determination of all the components of the current, the two perpendicular and the parallel ones. The average structure is shown in the lower panel of Fig. 2. It is clearly seen that the blobs appear as a filaments with a parallel component more than one order of magnitude greater than the perpendicular ones. As a further observation it can be noted that the parallel current perturbation exhibits a nearly monopolar structure in this case. The presence of a poloidal current in the RFP configuration is expected for the self -sustainment of the magnetic configuration itself, however further investigations are needed to reveal the current path of the filament.

The magnetic perturbations associated to this parallel current, determine fluctuations of the alfvén velocities, i.e. $\tilde{v}_{r}^{A}=\tilde{b}_{r} / \sqrt{\rho \mu_{0}}$ and $\tilde{v}_{\phi}^{A}=\tilde{b}_{\phi} / \sqrt{\rho \mu_{0}}$ which are found to be consistent with the measured perpendicular velocities ${ }^{20}$. Finally the direct comparison of 
Electromagnetic turbulent structures

parallel vorticity and parallel current shown in Fig. 3 (lower left panel) reveals that these two measurements are in phase one with the other and all these combined information has allowed the identification of these structures as Drift-Kinetic Alfvén vortices, as also observed for example in the magnetosphere ${ }^{2}$. It is worth mentioning that DKA was observed in discharges with reversal parameter $B_{\phi}(a) /\left\langle B_{\phi}\right\rangle \approx-0.07$. At different equilibria other driving mechanism could in principle generates these blobs, as for example pressure driven tearing modes which are found unstable at lower values of $q(a)^{36,37}$.

RFX-mod: Tokamak As aforementioned RFX-mod can operate also as a low-current circular ohmic tokamak. Data shown hereafter have been obtained during deuterium discharges at $I_{p} \approx 80 \mathrm{kA}$, with a $q_{95} \approx 3$. The data have been collected using the same diagnostic tools used for the RFX-mod RFP operation and refer to measurement at $r / a>0.9$, thus providing direct measurements of the electromagnetic features of filaments in the confined region of an L-mode ohmic tokamak. The diagnostic tool allows the determination of the $\mathbf{E} \times \mathbf{B}$ profile from potential measurements on a shot-to-shot basis. The result is shown in Fig. 3 in the top right. A sheared radial profile of the $v_{\mathbf{E} \times \mathbf{B}}$ main component,the poloidal one in this case, is observed. The measured shear is lower with respect to the RFP case as expected. Within the limited radial range explored the flow profile is consistent with the one already observed L-mode tokamak operation, however an higher shear is expected for $r / a \geq 0.98$ (see for example ${ }^{38}$ ). Intermittent blobs are revealed using as trigger event the appearance of intense fluctuations on the $p_{e}$ signal and applying the conditional average technique, as in the RFP case. As already done in the RFP case the fluctuations are identified at different time scales (through a continuous wavelet transform ${ }^{39}$ ) or correspondingly at different spatial scales. The results of this procedure are shown in figure 3 in the bottom right panel: the first fundamental observation is that the appearance of the blob is associated with an evident current structure. In this case, the current structure shape exhibits a main positive peak and two non-negligible negative peaks. The evaluation of the current structure integral provides a nearly zero value, so that we could hypothesize that the filament in this case closes the current loop on itself along the field line. Furthermore, as in the case of the RFP, a clear vorticity structure is associated with the current perturbation exhibiting a peak in phase with the maximum of the current. The results obtained confirm the EM feature of the blobs, also in the confined region of a L-mode tokamak, and also indicate that current and vorticity are two properties whose dynamics is intimately correlated. 
Electromagnetic turbulent structures

\section{B. TJ-II stellarator}

Stellarator TJ-II generally operates at lower beta value with respect to RFPs: however the TJ-II setup allow to explore clearly distinguishable density regimes within the same discharge. For the present experiment discharges were tailored with a limited maximum density, about $2.5 \times 10^{19} \mathrm{~m}^{-3}$, in order to allow a probe insertion radially up to $\rho=0.9$. A radial scan of the probe insertion was performed on a shot-to-shot basis. The typical discharge presented a first phase of $\sim 150 \mathrm{~ms}$ based on ECH heating system, with the plasma density limited to $0.5 \times 10^{19} \mathrm{~m}^{-3}$, in the second phase, approximately of the same duration, density increased up to $1.8 \times 10^{19} \mathrm{~m}^{-3}$ by NBI power injection. A second series of shots was then performed maintaining the probe at the maximum insertion and increasing the density at the maximum allowed value. In this way a not negligible variation of local beta was obtained within the shot. A sharp location of the LCFS is not available, however the most inserted measures have been obtained undoubtedly inside the confined region ${ }^{28}$. The time averaged radial profiles for the $E \times B$ poloidal flow, $v_{E \times B}$, and for electron pressure, $p_{e}$, are shown in figure 4. ECH and NBI phases are compared for each quantity. It is worth noting that opposite values of $v_{E \times B}$ and of its shear characterize the deepest position $(\rho<0.95)$. This feature can be imputed to the different density regimes rather than to the different heating system, in agreement with the results by Pedrosa et al. ${ }^{40}$. Regarding $p_{e}$ a steeper pressure gradient is observed during the NBI phase as expected.

ES blobs were already detected in TJ-II ${ }^{41}$ by imaging diagnostics. For the present study analogously to what performed in the RFX-mod, the investigation of presence and features of EM filaments in the TJ-II discharges was carried out by applying statistical methods based on wavelets ${ }^{30}$. Filaments events were then identified in the electrostatic fluctuation, i. e. fluctuation of density, and conditionally averaged on time windows of suitable amplitude in order to extract average properties of filaments . The procedure has been applied on different time intervals during the discharge, so that the average features of filaments could be analyzed also as a function of the corresponding plasma experimental conditions. In figure 5 the average features of filaments, triggered on $I_{\text {sat }}$ events are shown for density, temperature, vorticity and floating potential, during the ECH phase of the discharge. Different colors indicate different radial positions of the probe head. Filament structure averages are detected and associated to the presence of a density perturbation 
and to a cold temperature structure. The density perturbation is clearly associated to a $V_{f}$ structure and to a vorticity one. It is interesting to note that the vorticity structure, and the $V_{f}$ structure as well, shows a strong dependence on the radial position. Specifically the main vorticity peak exhibits a clear reduction moving towards deeper positions and it is worth noting that this region is characterized by an increasing positive shear with respect to the outer positions (see fig. 4). An analogous analysis has been carried out during the NBI phase, and is shown in figure 6. Again the filament density perturbation is associated to a structure in all the considered quantities, in particular a dipolar structure of $T_{e}$ is found analogously to what observed in RFX-mod ${ }^{8}$. Also in this case a clear average structure of vorticity is observed, however with the main vorticity peak increasing for deeper positions. It is worth recalling that the NBI phase is characterized by an opposite shear in the inner positions with respect to the ECH. It can be therefore concluded that the local flow shear affects the intrinsic vorticity of the structure through a process of polarity selection. This result further confirms the experimental observations in the RFP configuration ${ }^{39,42}$, where prograde and adverse vortex structures radially modulated accordingly to the local flow shear are also detected. This behavior is well assessed in fluid dynamics, and it is worth mentioning that recently ${ }^{43}$ it has been also considered from a theoretical point of view as the basis of non-diffusive transport mechanism in girokinetic simulations. As far as the EM average features of filament is concerned, the conditional average technique was applied to the measured $J_{\|}$. The applied analysis technique is the same described in the in RFX-mod case. As in the figures 5 and 6 the trigger for the identification of filaments is provided by the intermittent events observed in the $I_{\text {sat }}$ time trace. The average features of resulting EM filaments are compared in different plasma conditions for the insertion $\rho=0.9$. The explored experimental conditions are summarized in figure 7, where a local electron beta-main plasma density-local temperature graph is shown. On the right side the average perturbation in the $J_{\|}$signal due to the filament is shown for the higher local pressure, $p_{e}>8.5 \mathrm{~Pa}$, and for the lower values, $p_{e}<6 \mathrm{~Pa}$. The clearest sign of a $\delta J_{\|}$structure is observed for the highest pressure values, corresponding to the highest local electron beta, $\beta_{e}>0.002 \%$, whereas it disappears for the lowest $p_{e}$, corresponding to the $\beta_{e}<0.0016 \%$ and involving the extreme values of the discharge density explored. Focusing on the highest local $\beta_{e}$ case, it can be observed that the emerging parallel current density in the figure, upper panel in the right column of figure 7 , suggests a nearly dipolar structure. The integral of the current structure 
Electromagnetic turbulent structures

in the figure is nearly zero. This suggests, analogously to the RPX-mod tokamak case, that the filament current loop may close its path on itself along the local magnetic field line.

The role played by the local $\beta_{e}$ instead of a simple increase of density is confirmed by some dedicated experiments performed at even higher plasma density (up to $710^{19} / \mathrm{m}^{3}$ ) however not accompanied by an effective increase of beta, neither in these cases a clear evidence of parallel current associated to filamented structures was found.

\section{TORPEX Simple Magnetized Torus}

The TORPEX device features the plasma environment with the minimum $\beta_{e}$ among the cases treated. Here, measurements are performed in a Scrape Off Layer (SOL)-like region. It was experimentally proven ${ }^{44-46}$ that such a configuration, as predicted by theory and numerical simulations ${ }^{47}$, is dominated by an ideal interchange mode, localized at $r \simeq-3 \mathrm{~cm}$, around the region of maximum pressure gradient ${ }^{48-51}$. The experiment setup for the data-set shown here includes a conducting limiter, grounded on the vacuum vessel, which covers half of the poloidal section on the low field side of the torus, i.e. for $r>0$. The presence of the interchange mode and of the magnetic field curvature provide the drive to filamentary structures, which are generated by radially extending mode crests, from which they are sheared off due to the presence of a local bulk $E \times B$ flow $^{15,52-54}$. In the above-mentioned setup, blobs connected to both sides of the limiter are routinely observed. These filaments are elongated and parallel to the magnetic field lines and nearly perpendicularly incident onto the limiter surface with a connection length $L_{\|}=2 \pi R \approx 6 \mathrm{~m}$. The vertical displacement of a magnetic field line after one toroidal turn is $\triangle=2 \pi R B_{z} / B_{t}$ and corresponds to about $13 \mathrm{~cm}$. Owing to the milder plasma environment with respect to fusion oriented experiments, for the first time a complete cross-field 2D map of filament features was obtained in a SOL-like configuration in the limiter proximity, including a direct measurement of the parallel current density, $J_{\|}^{21}$. The result is shown in figure 8 , where the conditional averaged $2 \mathrm{D}$ profiles of different quantities measured at a distance of $3 \mathrm{~cm}$ from the limiter are presented. The trigger is provided by an ion saturation current signal, $I_{\text {sat }}$, measured $1 \mathrm{~m}$ away along the field line and the resulting density filament pattern is indicated by the black contours in fig. 8a. In the same figure, the 2D map of floating potential, $V_{f}$, is shown and exhibits a clear dipolar structure providing the experimental evidence of the charge separation acting as a drive for 
Electromagnetic turbulent structures

the filament currents, as required by the equivalent circuit model ${ }^{17}$. According to the above mentioned circuit scheme, filament parallel currents are allowed flowing towards the limiter and their existence is confirmed by the experimental maps in fig. 8b and c. In particular, they show the $2 \mathrm{D}$ maps of $J_{\|}$and of its fluctuating part $\delta J_{\|}$obtained respectively from the SSLP and from the L-shaped 'current probe'. With an excellent agreement, both diagnostics reveal a parallel current dipole associated with the filament, characterized by a pronounced asymmetry which was ascribed to the Bohm boundary conditions at the limiter ${ }^{55}$.

The new experimental challenge is represented by the 3D characterization of filaments EM features. Thanks to the high versatility of the TORPEX device and to its highly reproducible discharges, most of the 3D features associated to filaments can be obtained through a 2D map of the considered quantity in the cross-field plane and repeating the measurements at different toroidal positions. In particular, it is worth highlighting the presence of the limiter, which allows investigating clearly a paradigmatic experimental condition. The limiter introduces a well defined sheath region and this is the experimental condition assumed in the equivalent circuit model of a EM filaments.

The main tool used for the 3D characterization of ES filaments filaments is the probe dubbed 2DSSLP, which consists essentially of a 2D array of 8 Single-Sided Langmuir probes developed on a cross-field plane. The probe head is installed on a movable system spanning a 2D section on the radial-vertical plane. The conditional average method ${ }^{48}$ is applied for all the toroidal positions explored, and the density trigger is kept at a fixed position, $\left(r=7 \mathrm{~cm}, z=5.4 \mathrm{~cm}\right.$ and $\left.\phi=63^{\circ}\right)$, so that a 3D filament map can be obtained along the chosen magnetic field line. Measurements are performed on vertical sections on both side of the limiter, dubbed limiter side $A$ and limiter side $B$ respectively.

In figure $9 \mathrm{a}-\mathrm{c}$, the $2 \mathrm{D}$ maps of ion saturation current, $I_{\text {sat }}$, representative of the filament density pattern, of $V_{f}$ and of $J_{\|}$measured on the limiter side $A$ are shown. Analogous quantities are obtained on the other side of the limiter, side $B$, and shown in the d-f panels of the same figure. Comparing panels $\mathrm{c}$ and $\mathrm{f}$, the density pattern associated with the filament can be easily recognized on both sides of the limiter with a vertical displacement consistent with the field line displacement. The two opposite peaks detected on each one of the $V_{f}$ maps (panels b and e) confirm the presence of the charge separation.

Concerning $J_{\|}$fig. 9a and d, a strongly asymmetric current density structure associated to the filament is found. It is interesting to note that at both limiter sides an electron current 
Electromagnetic turbulent structures

density lobe placed on the bottom part of the filament (itself indicated by white contours) is observed, revealing that at both sides a dominant electron current density flows out from the limiter. Looking at the TORPEX torus from the top this means that the electron current, accompanying the density filament always on its bottom side, flows clockwise from the limiter side $A$, whereas it flows in the opposite direction from the limiter side $B$. As a consequence the current structure associated with the filament should change sign at a toroidal position along the selected magnetic field line, supposedly around L/2, where $\mathrm{L}$ is the connection length of the magnetic field line. These experimental observations, and in particular a "switch" of the current density dipole in the cross-field plane at $\mathrm{L} / 2$, are consistent with the theoretical expectations and provide, to our knowledge, the first experimental confirmation of the equivalent circuit of the current density filament associated to the blob and described in ${ }^{14,17}$. The filament characterization has been complemented also by the 3D map of the associated vorticity. The results are shown in figure 10(top panels) for three different toroidal positions moving from limiter side $A$ to side $B$. For each position the respective map of $V_{f}$ and of density pattern is shown in the bottom panel of fig. 10. It can be observed that $\omega_{\|}$ and $V_{f}$ maps are consistent, confirming the reliability of the diagnostic method. The 3D map reveals that the same vorticity pattern follows the blob along the whole magnetic field line confirming that the same charge separation drive acts all along the toroidal path, as expected from the unchanged relationship between the pressure gradient and B curvature, see also $^{15}$.

Summarizing the 3D measurements results it can be concluded that the EM filament exhibits a $\delta J_{\|}$odd-structure, whereas a $\delta \omega_{\|}$even-structure is found. The reported measurements represent a qualitative behavior of the 3D structure of different quantities associated to the plasma filament, in agreement with the theoretical expectations. A detailed quantitative investigation is still in progress.

\section{DISCUSSION: INTER-MACHINE COMPARISON}

The data presented up to now collect analogous information on filamentary structures through different devices and magnetic configurations allowing access to a wide range of plasma parameters and different magnetic topologies. Concerning this last aspect it has to be pointed out that in all the considered fusion experiments, TJ-II and RFX-mod, mea- 
Electromagnetic turbulent structures

surements were performed in the edge confined region, whereas in the TORPEX experiment measurements are in a SOL-like magnetic topology, due to the limiter configuration. The magnetic configurations investigated here, including open and closed field magnetic geometries, may result in different dominant instabilities and consequently distinct blob generation mechanisms. Furthermore, the local collisionality is expected to play an important role both on the lifetime and motion of the filaments ${ }^{56}$. A detailed investigation of the blob generation mechanism was carried out in the RFP ${ }^{20}$ and in the Torpex ${ }^{48}$ cases. Further investigations on the filaments generation mechanism will be the subject of future work. Presently, from the experimental point of view, we focused on the investigation on presence and features of EM filaments across different magnetic configurations and magnetic topologies within the aim of identifying possibly common physical features.

A first important observation is that in each configuration filamentary structures with EM features were found, it is interesting therefore to gain insights on their general properties from a direct quantitative comparison of the results throughout the explored configurations. As representative quantity of ES filament features can be considered its associated vorticity perturbation, $\delta \omega_{\|}$, or equivalently its vortex enstrophy $\left|\delta \omega^{2}\right|$. In order to explore a link with average local plasma properties, following the indication provided in figs 5 and $6, \delta \omega_{\|}$ has been plotted as a function of the time average local $E \times B$ flow shear. In figure 11, the peak-to-peak fluctuation of $\delta \omega_{\|}$is shown as a function of the local absolute value of the shearing rate. In all fusion devices, the data refers to the radial position $r / a \sim 0.94$. The result shows an increasing trend of $\delta \omega_{\|}$as the local shearing rate increases, covering at least two orders of magnitude. This behavior could be interpreted as the Biglary-DiamondTerry criterion ${ }^{57}$ extended to the filament parallel dynamics, complementing the picture of the blob cross-field size regulated by the shearing rate. According to the fig. 11, only high vorticity perturbations survive in a highly sheared region, or the other way round local shear favorites the prograde vortices. The local shear effect on filament vorticity structure could imply consequences on their related convective transport. The cross-field dipolar vorticity associated to the filaments plays an important role on their radial velocity. From statistical point of view in the RFP case it was reported that a radial distribution of dipolar structures is associated to the radial region characterized by the lower shear and higher diffusivity ${ }^{39}$. A direct observation of the filaments radial velocity of dipolar and monopolar filament vorticity could provide further information on this issue. However it has to be noted that generally 
Electromagnetic turbulent structures

the filaments are observed to travel according to the local $E \times B$ flow. This implies, in particular for the RFP case, that a direct observation of the radial velocity of filaments is experimentally difficult due to the highly dominant $E \times B$ toroidal velocity in the edge.

¿From the filament EM features point of view the ratio $\delta J_{\|} / B_{0}$ was considered as representative quantity, and its relationship with the local electron beta, $\beta_{e}$, is shown in figure 12 . A clear increase of $\delta J_{\|} / B_{0}$ with $\beta_{e}$ is observed. It is worth noting that the comparison of the different configurations allowed to explore about four order of magnitude in the $\beta_{e}$ parameter, so that it can be concluded that $\beta_{e}$ constitutes a key parameter ruling the EM features of filaments. These results could provide some experimental indications for the simulations of EM coherent structures.

These observations defintely represent a first attempt of providing an experimental scalings of EM filament features across different toroidal plasma machines. However a further effort is required for a deeper understanding of this behavior in particular from the theoretical point of view.

\section{CONCLUSIONS}

Analogous diagnostic methods have been applied across the different magnetic configurations explored, and successfully tailored to the respective plasma environments. The EM features of filamentary structures have then been detected and studied in all the considered devices. In particular a clear experimental confirmation of the filament equivalent circuit model $^{17}$, was obtained up to the 3D details in the SOL-like plasma, where filament parallel current is mainly regulated by Bohm boundary conditions.

Independently from the specific local magnetic topology, and given different drive mechanisms (DKA, ideal interchange) for the filaments formation, several common features are shared among the filaments detected in the different configurations explored both electrostatic, such as the presence of density and vorticity structures, and electromagnetic, with a parallel current density associated. More specifically the inter-machine comparison allows concluding that local plasma parameters seem to rule the intensity of some specific quantities and in particular the intensity of the vorticity associated to the filament exhibits a clear dependence on the local $E \times B$ shearing rate. Furthermore the relatively wide range of the local $\beta_{e}$ explored, allows concluding that this parameter play a fundamental role in the 
Electromagnetic turbulent structures

appearing of the EM features of the filaments. As a final remark the presented results could be relevant for simulation feedback of EM filaments and ultimately for providing hints for their experimental control.

\section{ACKNOWLEDGMENTS}

This project has received funding from the European Union's Horizon 2020 research and innovation programme under grant agreement number 633053. The views and opinions expressed herein do not necessarily reflect those of the European Commission.

\section{REFERENCES}

${ }^{1}$ A. Kirk, N. B. Ayed, G. Counsell, B. Dudson, T. Eich, A. Herrmann, B. Koch, R. Martín, A. Meakins, S. Saarelma, R. Scannell, S. Tallents, M. Walsh, H. R. Wilson, and t. M. team, Plasma Physics and Controlled Fusion 48, B433 (2006).

${ }^{2}$ D. Sundkvist, V. Krasnoselskikh, P. K. Shukla, A. Vaivads, M. André, S. Buchert, and H. Réme, Nature 436, 825 (2005).

${ }^{3}$ E. Martines, N. Vianello, D. Sundkvist, M. Spolaore, M. Zuin, M. Agostini, V. Antoni, R. Cavazzana, C. Ionita, M. Maraschek, F. Mehlmann, H. W. Müller, V. Naulin, J. J. Rasmussen, V. Rohde, P. Scarin, R. Schrittwieser, G. Serianni, E. Spada, t. R.-m. team, and t. A. U. Team, Plasma Physics and Controlled Fusion 51, 124053 (2009).

${ }^{4}$ B. A. Carreras, Plasma Science, IEEE Transactions on 25, 1281 (1997).

${ }^{5}$ D. A. D'Ippolito, J. R. Myra, and S. I. Krasheninnikov, Physics of Plasmas 9, 222 (2002).

${ }^{6}$ O. E. Garcia, Plasma and Fusion Research 4, 019 (2009).

${ }^{7}$ D. A. D'Ippolito, J. R. Myra, and S. J. Zweben, Physics of Plasmas 18, 060501 (2011).

${ }^{8}$ M. Spolaore, N. Vianello, M. Agostini, R. Cavazzana, E. Martines, P. Scarin, G. Serianni, E. Spada, M. Zuin, and V. Antoni, Physical Review Letters 102, 165001 (2009).

${ }^{9}$ I. Furno, C. Theiler, V. Chabloz, A. Fasoli, and J. Loizu, Physics of Plasmas (1994-present) 21, 012305 (2014).

${ }^{10}$ S. I. Krasheninnikov, D. D. Ryutov, and G. Yu, Journal Plasma Fusion Research SERIES 6, 139 (2004).

${ }^{11}$ N. Vianello, V. Naulin, R. Schrittwieser, H. W. Müller, M. Zuin, C. Ionita, J. J. Rasmussen, 
F. Mehlmann, V. Rohde, R. Cavazzana, and M. Maraschek, Physical Review Letters 106, $125002(2011)$.

${ }^{12}$ A. Wingen, T. E. Evans, C. J. Lasnier, and K. H. Spatschek, Physical Review Letters 104, $175001(2010)$.

${ }^{13}$ M. G. Dunne, P. J. McCarthy, E. Wolfrum, R. Fischer, L. Giannone, A. Burckhart, and the ASDEX Upgrade Team, Nuclear Fusion 52, 123014 (2012).

${ }^{14}$ J. R. Myra, Physics of Plasmas (1994-present) 14, 102314 (2007).

${ }^{15}$ C. Theiler, I. Furno, P. Ricci, A. Fasoli, B. Labit, S. H. Mueller, and G. Plyushchev, Physical Review Letters 103, 065001 (2009).

${ }^{16}$ C. Theiler, I. Furno, A. Fasoli, P. Ricci, B. Labit, and D. Iraji, Physics of Plasmas (1994-present) 18, 055901 (2011).

${ }^{17}$ S. I. Krasheninnikov, D. A. D'Ippolito, and J. R. Myra, Journal of Plasma Physics 74 (2008).

${ }^{18}$ B. Scott, Plasma Physics and Controlled Fusion 39, 1635 (1997).

${ }^{19}$ O. Grulke, S. Ullrich, T. Windisch, and T. Klinger, Plasma Physics and Controlled Fusion 49, B247 (2007).

${ }^{20}$ N. Vianello, M. Spolaore, E. Martines, R. Cavazzana, G. Serianni, M. Zuin, E. Spada, and V. Antoni, Nuclear Fusion 54, 042002 (2010).

${ }^{21}$ I. Furno, M. Spolaore, C. Theiler, N. Vianello, R. Cavazzana, and A. Fasoli, Physical Review Letters 106, 245001 (2011).

${ }^{22}$ C. Alejaldre, J. Alonso, L. Almoguera, E. Ascasíbar, A. Baciero, R. Balbín, M. Blaumoser, J. Botija, B. Brañas, E. de la Cal, A. Cappa, R. Carrasco, F. Castejón, J. R. Cepero, C. Cremy, J. Doncel, C. Dulya, T. Estrada, A. Fernández, M. Francés, C. Fuentes, A. García, I. García-Cortés, J. Guasp, J. Herranz, C. Hidalgo, J. A. Jiménez, I. Kirpitchev, V. Krivenski, I. Labrador, F. Lapayese, K. Likin, M. Liniers, A. López-Fraguas, A. LópezSánchez, E. de la Luna, R. Martín, A. Martínez, M. Medrano, P. Méndez, K. McCarthy, F. Medina, B. van Milligen, M. Ochando, L. Pacios, I. Pastor, M. A. Pedrosa, A. de la Peña, A. Portas, J. Qin, L. Rodríguez-Rodrigo, A. Salas, E. Sánchez, J. Sánchez, F. Tabarés, D. Tafalla, V. Tribaldos, J. Vega, B. Zurro, D. Akulina, O. I. Fedyanin, S. Grebenshchicov, N. Kharchev, A. Meshcheryakov, R. Barth, G. van Dijk, H. van der Meiden, and S. Petrov, Plasma Physics and Controlled Fusion 41, A539 (1999).

${ }^{23}$ A. Fasoli, A. Burckel, L. Federspiel, I. Furno, K. Gustafson, D. Iraji, B. Labit, J. Loizu, 
G. Plyushchev, P. Ricci, C. Theiler, A. Diallo, S. H. Mueller, M. Podestà, and F. Poli, Plasma Physics and Controlled Fusion 52, 124020 (2010).

${ }^{24}$ P. Sonato, G. Chitarin, P. Zaccaria, F. Gnesotto, S. Ortolani, A. Buffa, M. Bagatin, W. R. Baker, S. Dal Bello, P. Fiorentin, L. Grando, G. Marchiori, D. Marcuzzi, A. Masiello, S. Peruzzo, N. Pomaro, and G. Serianni, Fusion Engineering and Design 66-68, 161 (2003).

${ }^{25}$ P. Zanca, G. Marchiori, L. Marrelli, L. Piron, and t. R.-m. team, Plasma Physics and Controlled Fusion 54, 124018 (2012).

${ }^{26}$ J. A. Van Allen, American Journal of Physics 74, 809 (2006).

${ }^{27}$ H. Tsui, R. Bengtson, G. Li, H. Lin, M. Meier, C. P. Ritz, and A. Wootton, Review of scientific instruments 63, 4608 (1992).

${ }^{28}$ D. Carralero, Electromagnetic Instability Studies in Fusion Plasmas Edge, Ph.D. thesis, Universidad Politécnica de Madrid (2012).

${ }^{29}$ M. Spolaore, N. Vianello, M. Agostini, R. Cavazzana, E. Martines, G. Serianni, P. Scarin, E. Spada, M. Zuin, and V. Antoni, Journal of Nuclear Materials 390-391, 448 (2009).

${ }^{30}$ M. Farge, Annual Review of Fluid Mechanics 24, 395 (1992).

${ }^{31}$ V. Antoni, V. Carbone, E. Martines, G. Regnoli, G. Serianni, N. Vianello, and P. Veltri, EPL (Europhysics Letters) 54, 51 (2001).

${ }^{32}$ Pécseli, H L and Trulsen, J, Physica Scripta 43, 503 (1991).

${ }^{33}$ A. Nielsen, H. Pécseli, and J. J. Rasmussen, Physics of Plasmas (1994-present) 3, 1530 (1996).

${ }^{34}$ P. Scarin, M. Agostini, R. Cavazzana, F. Sattin, G. Serianni, M. Spolaore, and N. Vianello, Journal of Nuclear Materials 390-391, 444 (2009).

${ }^{35}$ U. Frisch, Turbulence: The Legacy of A. N. Kolmogorov (Cambridge University Press, 1996).

${ }^{36}$ R. Paccagnella, Physics of Plasmas 20, 012119 (2013).

${ }^{37}$ M. Zuin, S. Spagnolo, R. Paccagnella, E. Martines, R. Cavazzana, G. Serianni, M. Spolaore, and N. Vianello, Nuclear Fusion 50, 052001 (2010).

${ }^{38}$ G. D. Conway, C. Angioni, F. Ryter, P. Sauter, J. Vicente, and ASDEX Upgrade Team, Physical Review Letters 106, 065001 (2011).

${ }^{39}$ M. Spolaore, V. Antoni, E. Spada, H. Bergsåker, R. Cavazzana, J. R. Drake, E. Martines, G. Regnoli, G. Serianni, and N. Vianello, Physical Review Letters 93, 215003 (2004). 
${ }^{40}$ M. A. Pedrosa, C. Hidalgo, E. Calderón, T. Estrada, A. Fernández, J. Herranz, I. Pastor, and t. T.-I. team, Plasma Physics and Controlled Fusion 47, 777 (2005).

${ }^{41}$ J. A. Alonso, S. J. Zweben, P. Carvalho, J. L. de Pablos, E. de la Cal, C. Hidalgo, T. Klinger, B. P. van Milligen, R. J. Maqueda, M. Pedrosa, C. Silva, H. Thomsen, and M. Spolaore, Plasma Physics and Controlled Fusion 48, B465 (2006).

${ }^{42}$ M. Spolaore, V. Antoni, R. Cavazzana, G. Regnoli, G. Serianni, E. Spada, N. Vianello, H. Bergsåker, and J. R. Drake, Physics of Plasmas 9, 4110 (2002).

${ }^{43}$ R. Sánchez, D. E. Newman, J. N. Leboeuf, and V. K. Decyk, Plasma Physics and Controlled Fusion 53, 074018 (2011).

${ }^{44}$ P. Ricci, C. Theiler, A. Fasoli, I. Furno, K. Gustafson, D. Iraji, and J. Loizu, Physics of Plasmas (1994-present) 18, 032109 (2011).

${ }^{45}$ F. M. Poli, S. Brunner, A. Diallo, A. Fasoli, I. Furno, B. Labit, S. H. Müller, G. Plyushchev, and M. Podesta, Physics of ... 13, 102104 (2006).

${ }^{46}$ F. M. Poli, P. Ricci, A. Fasoli, and M. Podesta, 15, 032104 (2008).

${ }^{47}$ P. Ricci and B. N. Rogers, Physical Review Letters 104, 145001 (2010).

${ }^{48}$ I. Furno, B. Labit, M. Podestà, A. Fasoli, S. Müller, F. Poli, P. Ricci, C. Theiler, S. Brunner, A. Diallo, and J. Graves, Physical Review Letters 100, 055004 (2008).

${ }^{49}$ M. Podestà, A. Fasoli, B. Labit, M. McGrath, S. H. Müller, and F. M. Poli, Plasma Physics and Controlled Fusion 47, 1989 (2005).

${ }^{50}$ A. Diallo, A. Fasoli, I. Furno, B. Labit, M. Podestà, and C. Theiler, Physical review ... 101, 115005 (2008).

${ }^{51}$ C. Theiler, A. Diallo, A. Fasoli, I. Furno, B. Labit, M. Podestà, F. M. Poli, and P. Ricci, Physics of Plasmas (1994-present) 15, 042303 (2008).

${ }^{52}$ I. Furno, B. Labit, A. Fasoli, F. M. Poli, P. Ricci, and C. Theiler, Physics of Plasmas 15, 055903 (2008).

${ }^{53}$ S. H. Müller, A. Diallo, A. Fasoli, I. Furno, B. Labit, and M. Podestà, Physics of Plasmas (1994-present) 14, 110704 (2007).

${ }^{54}$ S. H. Müller, C. Theiler, A. Fasoli, I. Furno, B. Labit, G. R. Tynan, M. Xu, Z. Yan, and J. H. Yu, Plasma Physics and Controlled Fusion 51, 055020 (2009).

${ }^{55}$ I. Furno, C. Theiler, D. Lancon, A. Fasoli, D. Iraji, P. Ricci, M. Spolaore, and N. Vianello, Plasma Physics and Controlled Fusion 53, 124016 (2011).

${ }^{56}$ G. Xu, V. Naulin, W. Fundamenski, J. J. Rasmussen, A. H. Nielsen, and B. Wan, Physics 
Electromagnetic turbulent structures

of Plasmas (1994-present) 17, 022501 (2010).

${ }^{57}$ H. Biglari, P. H. Diamond, and P. W. Terry, Physics of Fluids B: Plasma Physics (19891993) 2, 1 (1990).
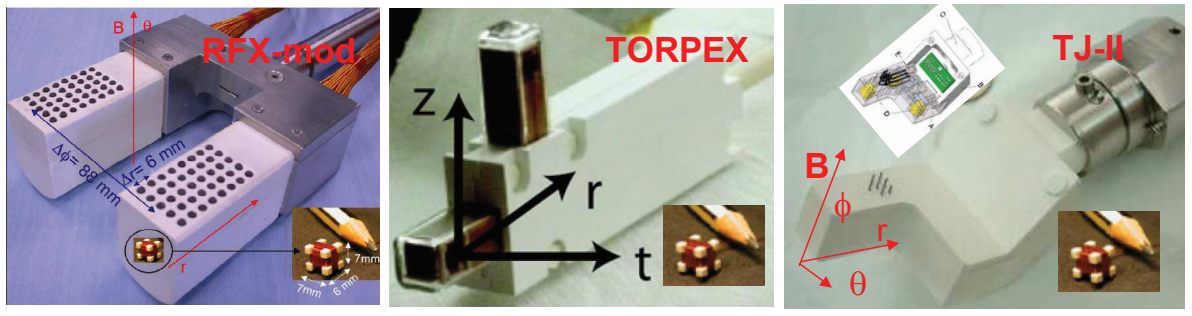

FIG. 1: The three panels show the diagnostics tools specifically designed for the investigation of the em filament structures. From left to right are shown respectively: the U-probe used in RFX-mod, the L-cluster probe used in Torpex, the vorticity probe used in TJ-II. The small square in each of the pictures shows one of the 3-axial magnetic probes embedded in the probe heads. 
Electromagnetic turbulent structures

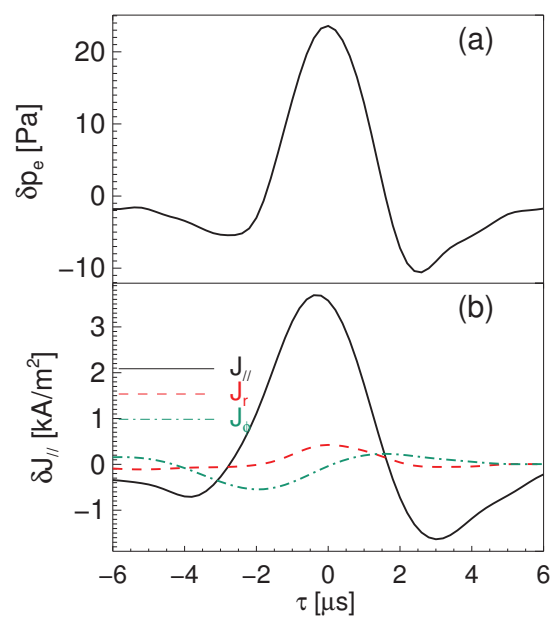

FIG. 2: RFX-mod RFP: pressure perturbation (a) and parallel and transverse components of $\delta J$ associated to a filament, conditional average on intermittent events detected on pressure signal at $200 \mathrm{kHz}$ time scale.
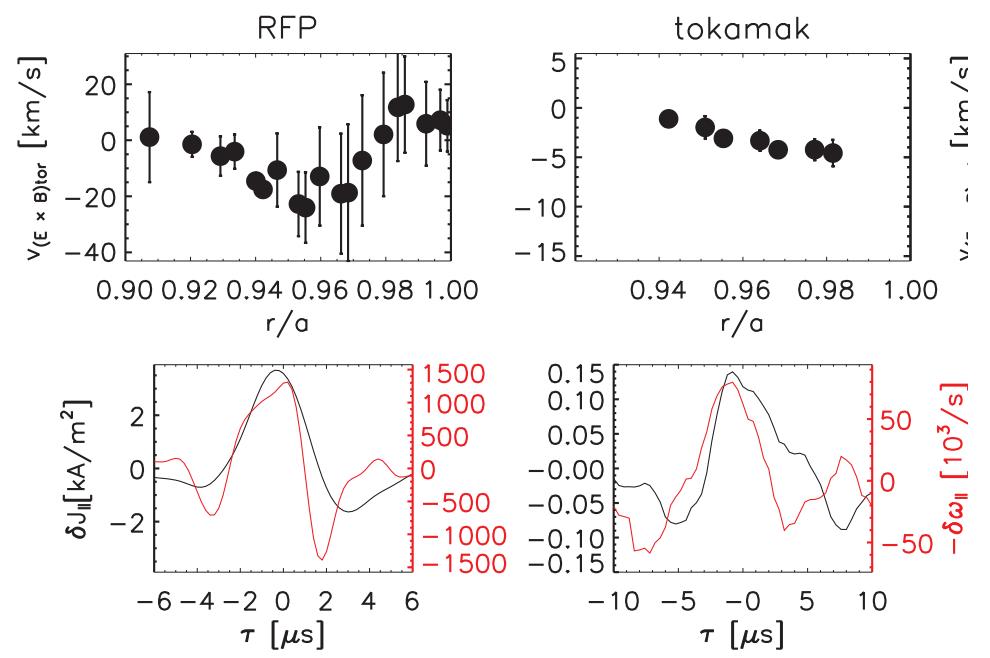

FIG. 3: Top panels: radial average profiles of $\mathbf{E} \times \mathbf{B}$ flow in RFX-mod. Toroidal component for RFP configuration (reversal $F>-0.05$ )(left); poloidal one for tokamak (q(a)=2.9)

(right). Bottom panels: conditional average of parallel current density and parallel vorticity structures detected at $200 \mathrm{kHz}$ in $\mathrm{RFP}$, trigger is provided by the intermittent events in local pressure(left); conditional average of parallel current density and the parallel vorticity associated to structures detected at $250 \mathrm{kHz}$ on tokamak discharge, the filament trigger is provided by the the intermittent events in local pressure (right). 
Electromagnetic turbulent structures

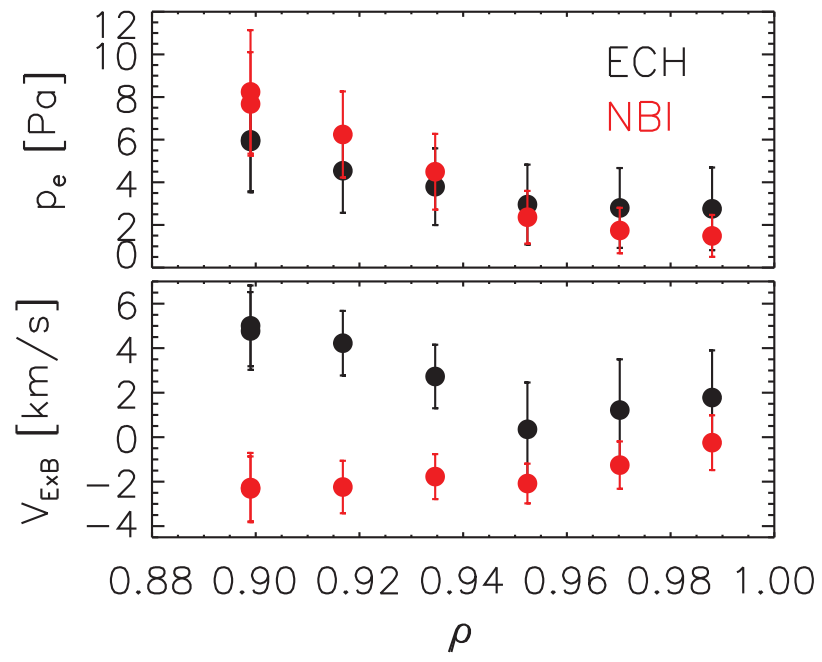

FIG. 4: TJ-II: average radial profile of $p_{e}$ and $v_{E \times B}$ in the poloidal direction, measured during ECH phase (black dots) and during NBI phase (red dots). 

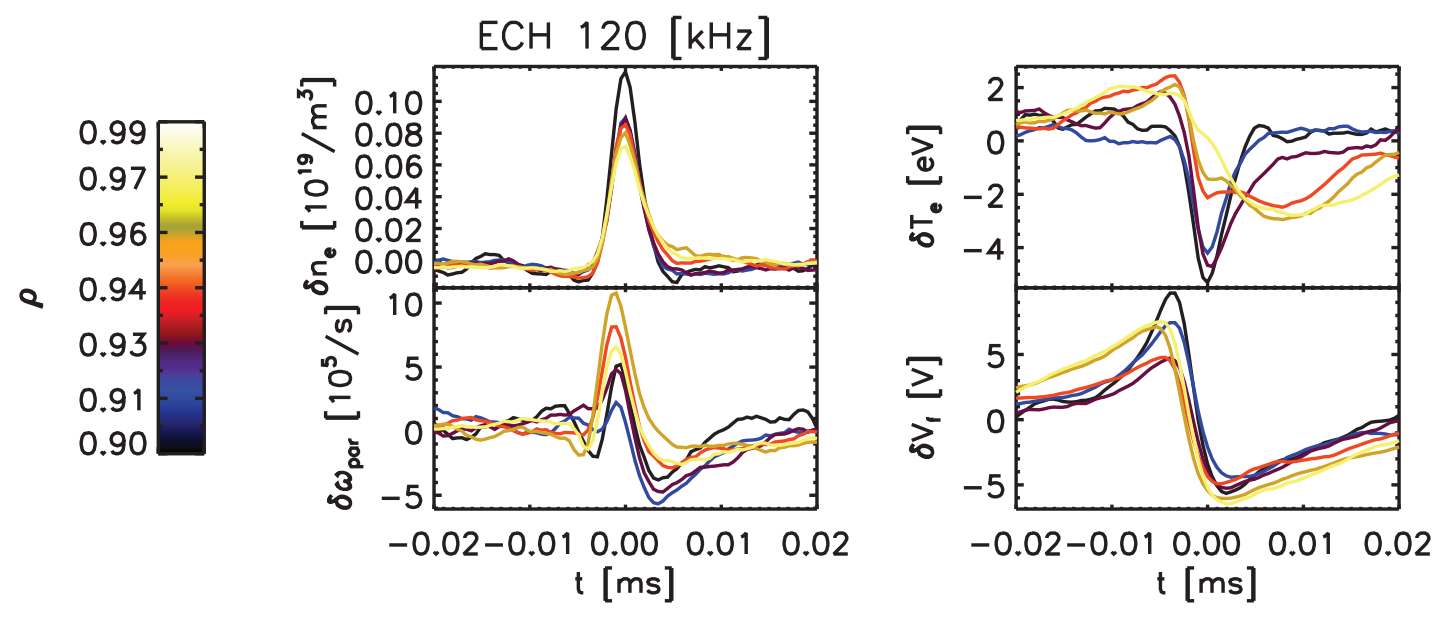

FIG. 5: TJ-II: Conditional average structure of fluctuating quantities (density, temperature, vorticity and floating potential), obtained with a trigger on $I_{\text {sat }}$ intermittent events detected at a time scale of $120 \mathrm{kHz}$. The panels show structures obtained during the ECH phase. Different colors indicate the structure features as obtained for different probe radial position $(\rho)$ following the color-code in the left colorbar. 
Electromagnetic turbulent structures
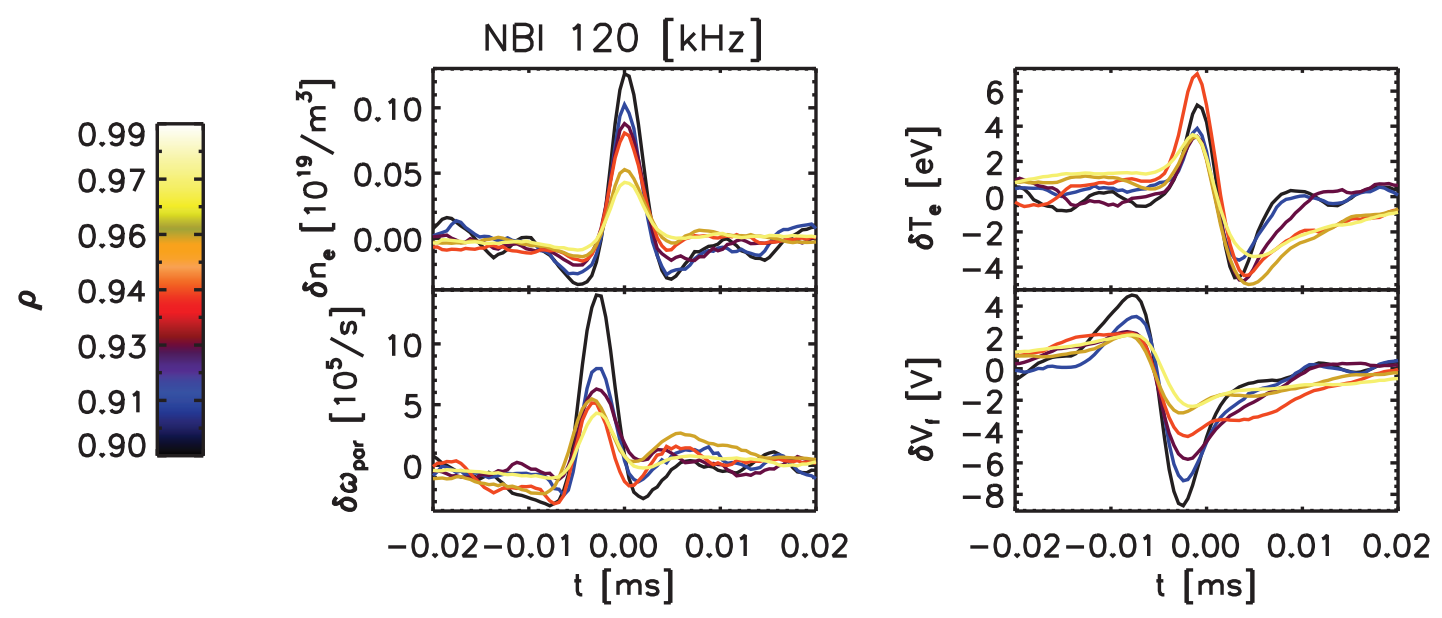

FIG. 6: The same as in figs 5 for the NBI plasma phase in TJ-II. 
Electromagnetic turbulent structures
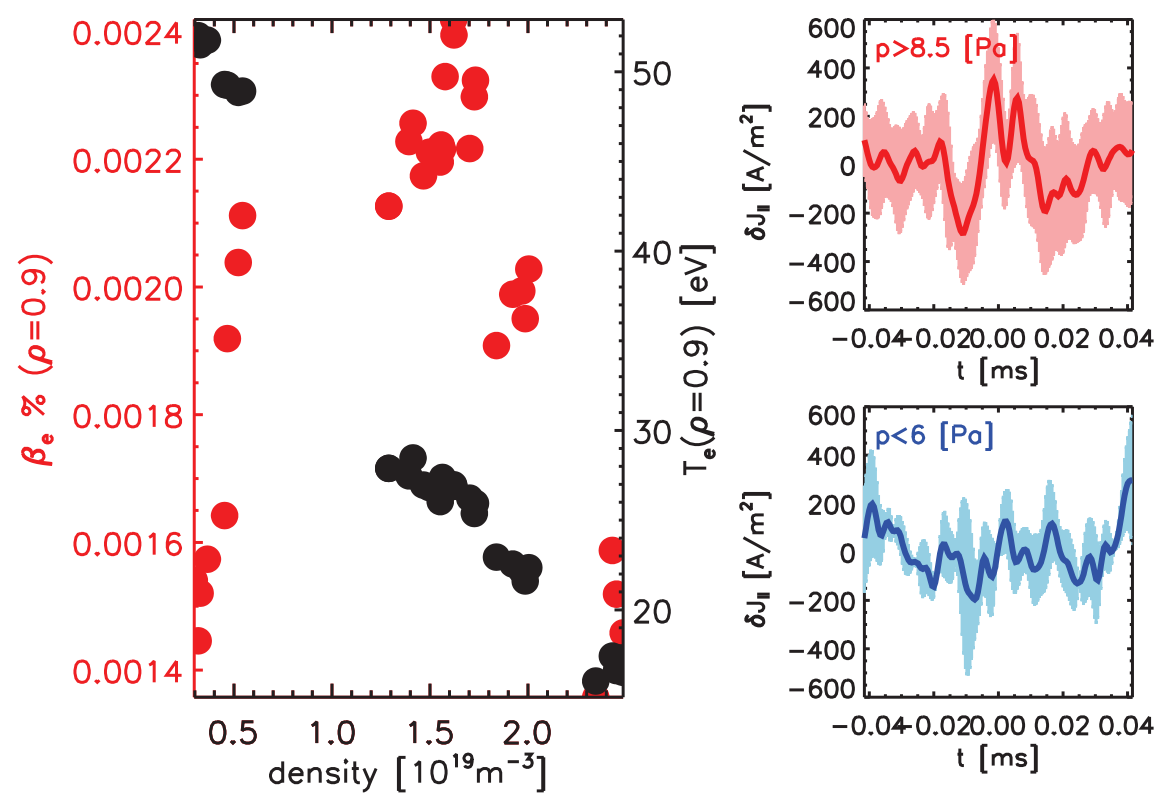

FIG. 7: TJ-II: summary of the explored experimental conditions in terms of central density, electron temperature and local electron beta (left panel); on the right column the resulting conditional average of $\delta J_{\|}$structure averaged over two extreme classes of the local electron pressure. The conditional average current density structures are obtained with a trigger on $I_{\text {sat }}$ intermittent events detected at a time scale of $120 \mathrm{kHz}$. 
Electromagnetic turbulent structures

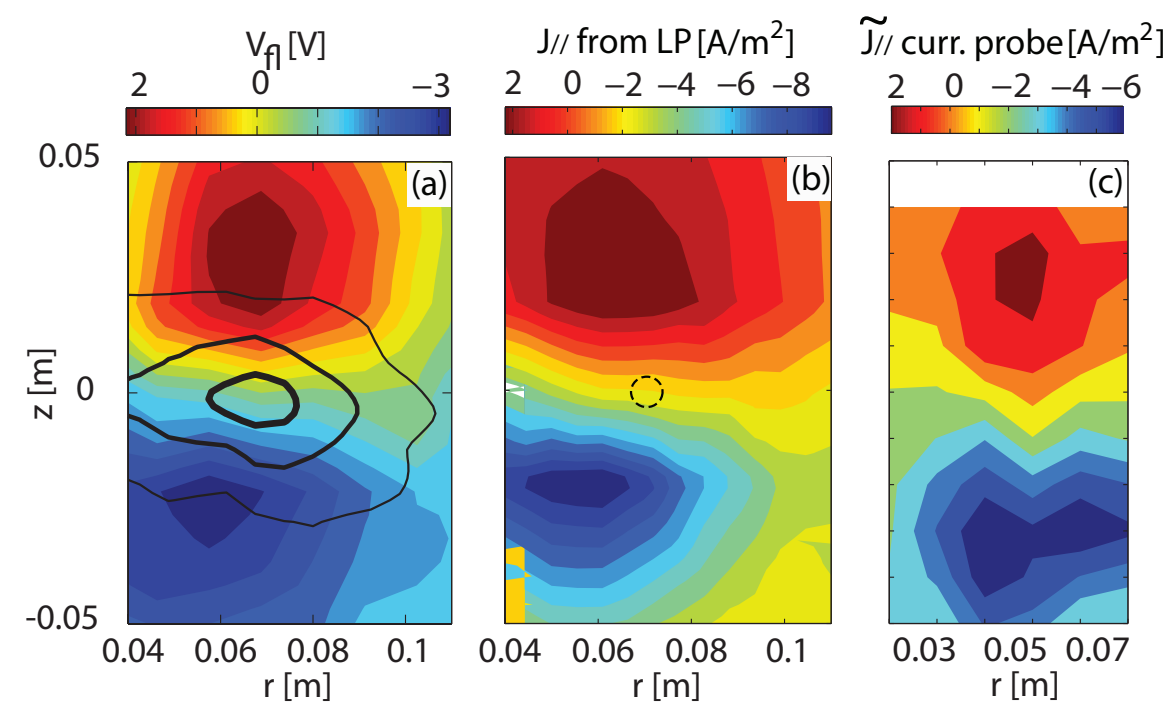

FIG. 8: Conditionally averaged 2D map of different quantities associated to a filament in the $(\mathrm{r}, \mathrm{z})$ plan, measured $\sim 3 \mathrm{~cm}$ toroidally apart from the limiter, the trigger is provided by $I_{\text {sat }}$ events measured $1 \mathrm{~m}$ away in the same direction: (a) floating potential, $V_{f}$, and position of filament density (black contours); (b) $J_{\|}$as measured by single-sided LP; (c) $\widetilde{J}_{\|}$ provided by the current probe.

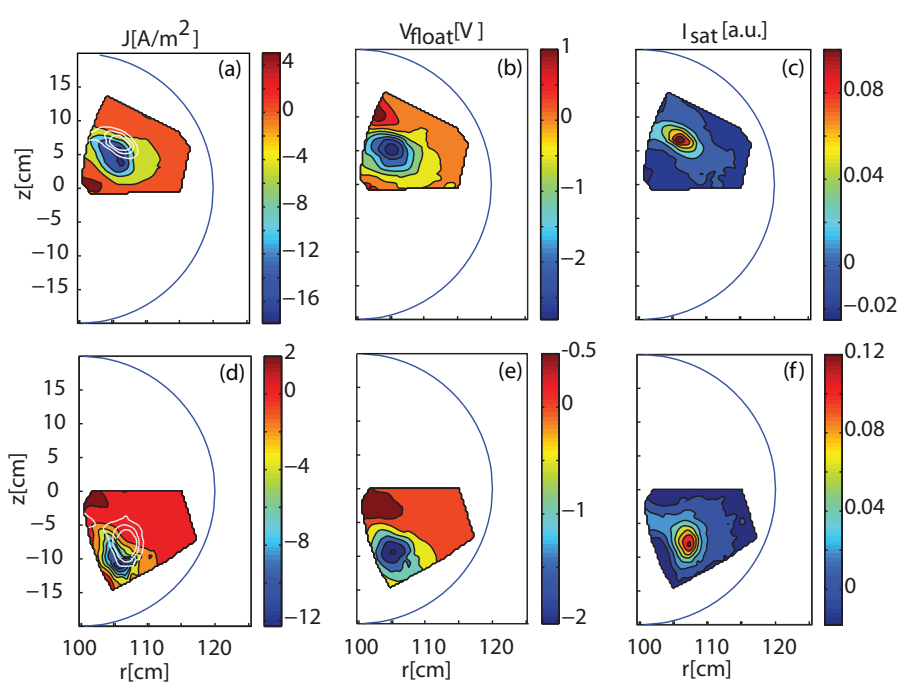

FIG. 9: Conditionally averaged 2D filament maps obtained using 2DSSLP. Shown are current density (a) and (d), Vf (b) and (e), and Isat (c) and (f). The filament $I_{\text {sat }}$ map is also represented by white contours in (a) and (b). Trigger is provided by $I_{\text {sat }}$ events detected with SLP at $r=7 \mathrm{~cm}, z=5.4 \mathrm{~cm}$ at $\pi / 7$ toroidally from limiter side A. Panels $(a)-(c)$ and $(d)-(f)$ refer to limiter side A and B respectively. 
Electromagnetic turbulent structures
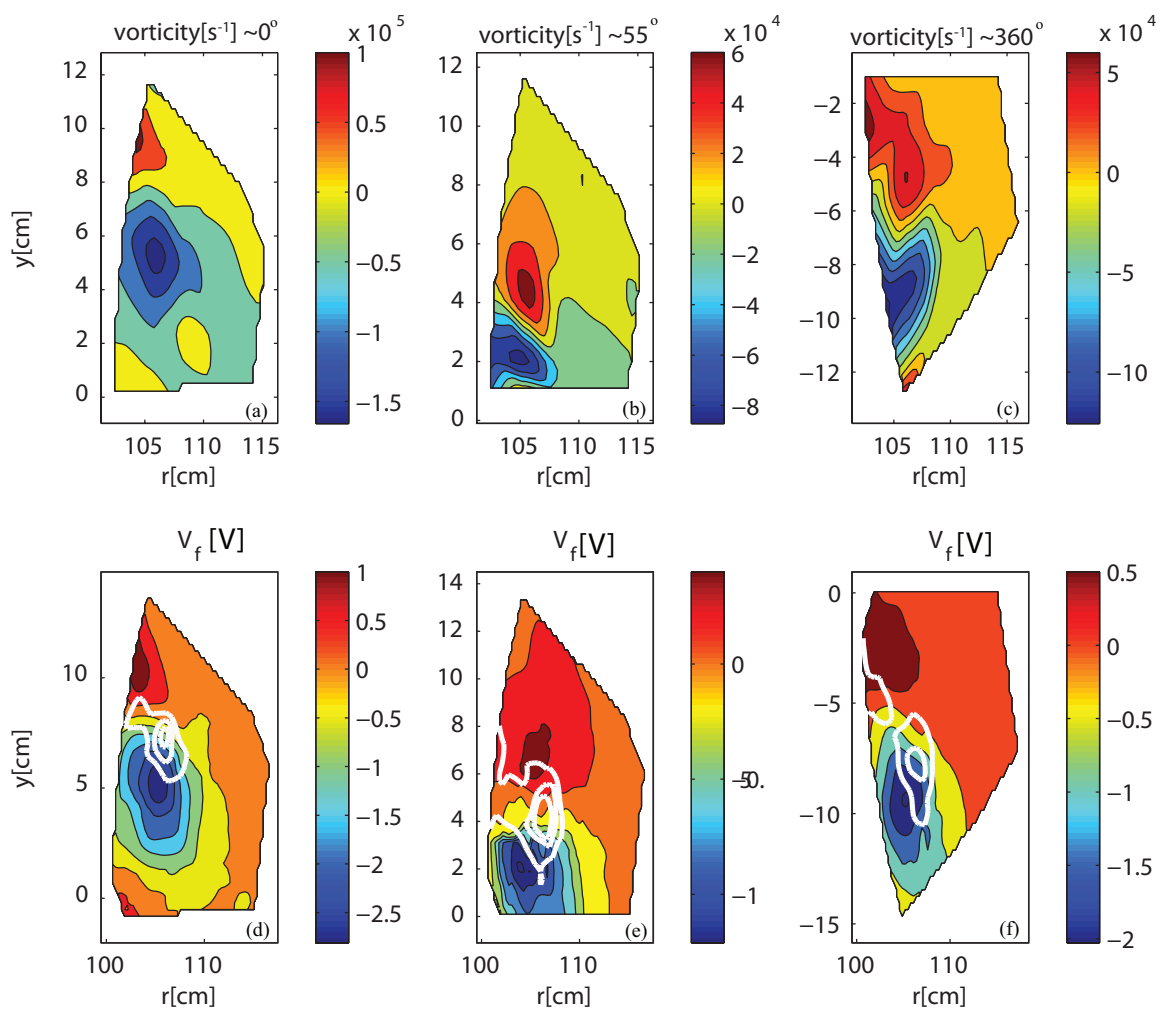

FIG. 10: Conditionally averaged 2D map of $V_{f}(\mathrm{~d})$-(f) measured in three different toroidal sections $\left(0^{\circ}\right.$ corresponds to the limiter side A). The white contour indicate the respective position of the filament density structure. Panels (a)-(c) show the corresponding 2D maps of the vorticity, data from 2DSSLP.

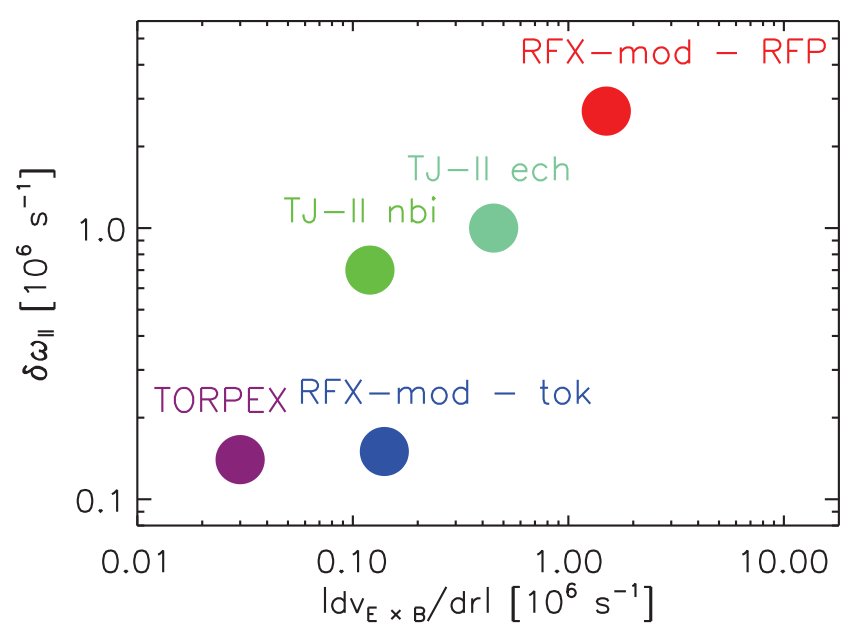

FIG. 11: Vorticity associated to structure vs local average $E \times B$ flow shear. 
Electromagnetic turbulent structures

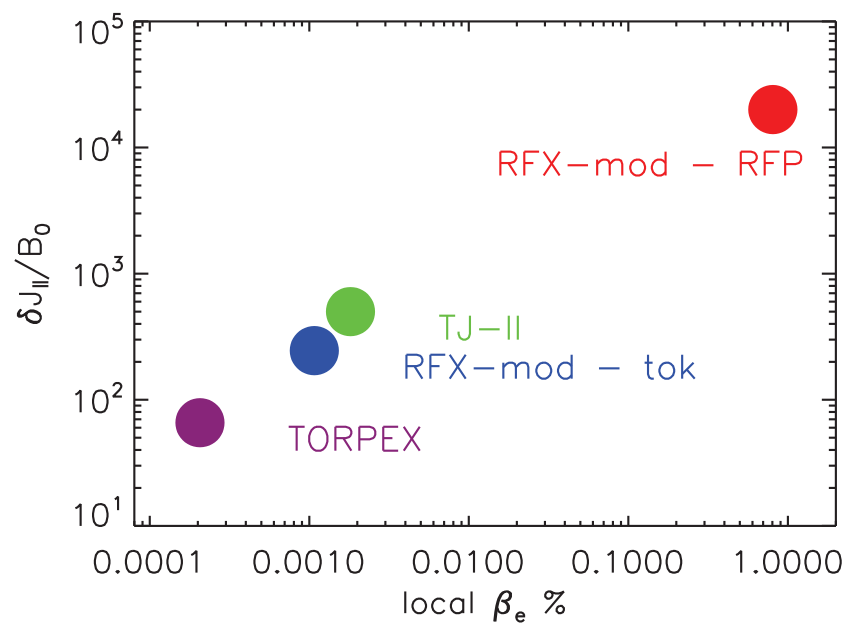

FIG. 12: $\delta J_{\|} / B_{0}$ vs local electron beta. 